\title{
Diseño de una secuencia de enseñanza-aprendizaje para el desarrollo de competencias científicas en el contexto del consumo de agua envasada
}

\author{
Francisco Rodríguez Mora \\ Instituto Provincial de Educación Permanente de Málaga. \\ Didáctica de las Ciencias Experimentales. Universidad de Málaga.España.franrodriguez@uma.es \\ Ángel Blanco López iD \\ Didáctica de las Ciencias Experimentales. Universidad de Málaga. España.ablancol@uma.es
}

[Recibido: 16 septiembre 2020. Revisado: 23 noviembre 2020. Aceptado: 9 diciembre 2020]

\begin{abstract}
Resumen: Se describe el proceso de elaboración de una secuencia de enseñanza-aprendizaje para el desarrollo de competencias científicas en el contexto del consumo de agua envasada, siguiendo los planteamientos de la investigación basada en el diseño. Después de los estudios preliminares, en torno al análisis didáctico del problema escogido y a la exploración de las ideas y creencias de los estudiantes sobre el mismo, se llevaron a cabo tres ciclos de diseño, implementación y análisis de diferentes versiones de la secuencia de enseñanza-aprendizaje, en la que participaron 68 estudiantes de $3^{\circ}$ y $4^{\circ}$ de la Educación Secundaria Obligatoria. Como resultado del proceso se llegó a una versión de la secuencia que ha permitido integrar un buen número de elementos provenientes de diversos campos: a) aspectos relevantes sobre el consumo de agua de bebida (del grifo y envasada); b) algunas ideas y creencias significativas sobre el consumo de agua de bebida, al objeto de contrastarlas con el conocimiento científico; c) conocimientos (de ciencia y sobre ciencia) necesarios para comprender adecuadamente los aspectos considerados sobre el consumo de agua de bebida; y d) el desarrollo de competencias científicas. Finalmente se presentan algunas consideraciones sobre los resultados obtenidos, los aprendizajes y la percepción de los estudiantes sobre la secuencia; así como la aportación de esta investigación a la enseñanza para el desarrollo de competencias científicas en el contexto de problemas y situaciones de la vida diaria.
\end{abstract}

Palabras clave: Competencias científicas, Enseñanza en contexto, Consumo de agua envasada, Secuencia de enseñanza-aprendizaje, Investigación basada en el diseño.

Design of a teaching-learning sequence for the development of scientific competences in the context of bottled water consumption

Abstract: The design process of a teaching-learning sequence for the development of scientific competences in
the context of bottled water consumption, following the approaches of design-based research is described. After
the preliminary studies, around the educational analysis of the chosen problem and the exploration of students'
ideas and beliefs about it, three cycles of design, implementation and analysis of different versions of the
teaching learning sequence were carried out- learning, in which 68 grades 9 and 10 students of Compulsory
Secondary Education participated. As a result of the process, a teaching-learning sequence version was reached
that has allowed the integration of a good number of elements from various fields: a) relevant aspects of
drinking water consumption (tap and bottled); b) some significant ideas and beliefs about drinking water
consumption, in order to contrast them with scientific knowledge; c) knowledge (scientific and about science)
necessary to understand the aspects considered on the consumption of drinking water; and d) the development
of scientific competences. Finally, some considerations are presented about the results obtained, on students
learning and students' perception about the sequence; as well as the contribution of this research to teaching for
the development of scientific competences in the context of problems and situations of daily life.

Keywords: Scientific competences, Context-based science teaching, Consumption of bottled water, Teaching-learning sequence, Designbased research.

Para citar este artículo: Rodríguez Mora F. y Blanco López A. (2021) Diseño de una secuencia de enseñanzaaprendizaje para el desarrollo de competencias científicas en el contexto del consumo de agua envasada. Revista Eureka sobre Enseñanza y Divulgación de las Ciencias 18(1), 1803. doi: 10.25267/Rev_Eureka_ensen_divulg_cienc.2021.v18.11.1803 


\section{Introducción}

Las situaciones y contextos de la vida diaria se vienen utilizando desde hace varias décadas como un importante recurso para la enseñanza de las ciencias (Bennet, Lubben y Hogarth 2007) con la finalidad de poner de manifiesto la relevancia del conocimiento científico, lo que ayuda a aumentar el interés de los estudiantes por su formación científica y favorecer una actitud más positiva hacia la ciencia (Caamaño 2005). Estos aspectos cobran especial atención con la llegada del enfoque educativo basado en el desarrollo de competencias clave para el aprendizaje permanente (Sanmartí 2008) y con la evaluación de competencias científicas en el programa PISA (OECD 2006).

La inclusión de competencias en los currículos oficiales (MEC 2006) tiene varias finalidades, entre ellas la de permitir a los estudiantes integrar sus aprendizajes, ponerlos en relación con distintos tipos de contenidos y utilizarlos de manera efectiva cuando les resulten necesarios en diferentes situaciones y contextos (Sanmartí 2008). Ahora bien, resulta imposible en las clases de ciencias abordar todos los contextos que se conocen en la actualidad, por lo que su elección constituye una fase crítica en el proceso de diseño y desarrollo curricular (Blanco, España y Rodríguez Mora 2012). Un criterio a considerar es el de la relevancia para la vida diaria del estudiante (en los ámbitos personal, social o global) de tal forma que su aprendizaje constituya un fin en sí mismo y que, a su vez, representen una oportunidad para construir ideas clave de la ciencia y sus interrelaciones (los modelos teóricos) (Marchán y Sanmartí 2015).

En esta investigación partimos de la premisa de que el «consumo de agua de bebida envasada» puede brindar un conjunto de situaciones y problemas de interés con potencial educativo, esto es, relevante y adecuado para constituir el contexto de secuencias de enseñanza-aprendizaje (en adelante SEAs). Ciertas controversias asociadas con el consumo de agua de bebida envasada como alternativa al agua de la red pública (agua del grifo) son consideradas de interés por parte de la ciudadanía, con importantes repercusiones en diferentes aspectos de la vida diaria; por ejemplo, en el ámbito de la salud (Ferrier 2001), pero también a nivel social con importantes implicaciones económicas y medioambientales (Gleick y Cooley 2009).

Este trabajo pretende contribuir a una doble exigencia, esto es, el diseño de una SEA que utilice el contexto y los conocimientos de ciencia como eje central para estructurar las actividades de enseñanza-aprendizaje y, a su vez, integrarlos con el tratamiento de competencias científicas. Basándonos en estos planteamientos, en este artículo se presenta el diseño y desarrollo de una SEA centrada en «el consumo de agua de envasada» para estudiantes de Educación Secundaria Obligatoria (ESO), siguiendo los planteamientos de la investigación basada en el diseño (en adelante IBD) (DBRC 2003).

\section{Fundamentos teóricos}

\section{El diseño de SEAs y el desarrollo de competencias}

Un elemento clave del diseño y la intervención educativa lo constituye la SEA, entendida como un documento para la planificación específica de las situaciones de enseñanza y aprendizaje correspondiente a un tema o contenido disciplinar concreto. Como herramienta básica de planificación una SEA debe ofrecer respuestas a cuestiones tan importantes como qué contenidos concretos enseñar, en qué contexto, con qué objetivos, en qué orden, de qué forma se llevan a cabo y se evalúan las actividades que se realizan, etc., además de incluir los materiales y recursos que se van a utilizar (Couso 2011).

Desde la década de los años 80 del pasado siglo el diseño e implementación de SEAs, constituye también un importante campo de, y para, la investigación educativa (Méheut y Psillos 2004) intentando relacionar el contenido disciplinar con los intereses y perspectivas de 
los estudiantes. Desde esta reciente y más amplia perspectiva, el concepto de SEA engloba aquellas propuestas didácticas que constan de un conjunto articulado de actividades de enseñanza y aprendizaje basadas en la investigación, probadas y adaptadas al nivel evolutivo y a las pautas de aprendizaje esperadas de los estudiantes (Buty, Tiberghien y Le Maréchal 2004). En otras palabras, representa un producto soportado en los resultados de la investigación didáctica, tanto de las concepciones de los estudiantes como de los contenidos de ciencia, a la vez que el propio diseño, su implementación y validación constituyen contextos para el desarrollo de investigación educativa acerca de la enseñanza y aprendizaje de la ciencia (DBRC 2003), e incluso, como un campo de investigación suficientemente relevante para modificar los procesos de enseñanza y aprendizaje (Méheut y Psillos 2004).

Construir y planificar SEAs para enseñar ciencias implica, entre otros aspectos, seleccionar cuidadosamente las actividades de enseñanza-aprendizaje que se consideran más adecuadas para las finalidades que se proponen (Marchán y Sanmartí 2015). En este sentido, si contemplamos el desarrollo de competencias científicas como las finalidades educativas, tal y cómo se plantean en los currículos actuales (MECD 2013), necesitamos partir de un enfoque explícito y claramente formulado sobre cómo entender las competencias científicas para poder vincularlas con las tareas y actividades de enseñanza-aprendizaje (Franco, Blanco y España 2017). Esto requiere identificar y precisar qué aspectos concretos de dichas competencias se van a poner en juego en las actividades que se diseñan y cómo se integran con las ideas científicas que los estudiantes tienen que aprender (Tekkumru-Kisa, Stein y Schunn 2015).

\section{El consumo de agua envasada como contexto de enseñanza-aprendizaje}

Un problema que afecta a los ciudadanos, en particular en los países desarrollados, es el de decidir de manera responsable y fundamentada qué tipo de agua de bebida es mejor consumir, del grifo o envasada: la controversia ya viene de lejos (Ferrier 2001). Necesitamos agua potable para que nuestro organismo funcione correctamente, pero muchos ciudadanos suelen dudar, con razón o no, de la seguridad del agua de abastecimiento público, considerada como un tipo de agua «poco saludable». Las continuas campañas de marketing por parte de las empresas embotelladoras y la influencia de la publicidad, entre otros factores, han hecho que de manera importante muchos ciudadanos aumenten su interés por el agua de consumo envasada. De esta forma, un producto de consumo, que apenas existía hace unas pocas décadas, se ha convertido en un elemento casi indispensable de nuestro modo de vida, que los consumidores perciben como una alternativa de superior calidad al agua ordinaria que podemos obtener del grifo (Da Cruz 2006, Ferrier 2001, Royte 2008). En paralelo a este crecimiento aumenta también las controversias sobre su empleo, motivo de importantes polémicas, debates y conflictos entre los distintos agentes implicados: ciudadanos, investigadores, empresas embotelladoras (Gleick y Cooley 2009). Estas controversias tienen relación con el cuidado de la salud (Shotyc, Krachler y Chen 2006), aspectos socioeconómicos (Wilk 2006), impacto social y cultural, y cuestiones medioambientales, políticas y éticas (Ferrier 2001). Los aspectos citados requieren por parte de los ciudadanos la toma de decisiones, conformando un escenario donde el acceso a la información, la ciencia y la tecnología juegan un papel importante (Jiménez y Otero 2012) y en cuya toma de conciencia se involucran los valores, las actitudes y creencias de los consumidores (Rodríguez Mora y Blanco 2009 y 2012). Por todas estas razones, consideramos que el consumo de agua envasada constituye un contexto relevante para su utilización en la enseñanza de las ciencias en la educación obligatoria (Blanco y Rodríguez Mora 2008).

El agua de bebida también se ha utilizado como eje central para distintas propuestas didácticas elaboradas desde la perspectiva de la alfabetización científica (Gavidia 2009 y Marcén 2009), al integrar distintos conocimientos de química con el tratamiento de aspectos relevantes 
relacionados con el consumo de agua: origen del agua que bebemos, tratamiento y desinfección del agua potable de la red pública, vigilancia sanitaria del agua de bebida, tipos de agua, etc. (Pryde et al. 2005). Como señalan Marcén y Cuadrats (2012) son muchos los argumentos para enseñar y aprender sobre el agua en la enseñanza obligatoria.

No obstante, hay que resaltar que el consumo de agua envasada es una cuestión que ha sido poco tratada desde la perspectiva que se pretende en esta investigación. Abordar este problema didáctico se convierte en la finalidad general de la investigación en la que se inserta este trabajo, y que podríamos concretar en los siguientes términos: ¿cómo integrar en una SEA el tratamiento de aspectos concretos del consumo de agua de bebida, el desarrollo de competencias científicas por parte de los alumnos y la construcción de conocimiento científico acerca de las disoluciones?

\section{Metodología}

Desde el punto de vista metodológico en este trabajo se han seguido los planteamientos de la investigación basada en el diseño (IBD) (DBRC 2003). Bajo la expresión «investigaciones de diseño» se engloba un amplio espectro de enfoques de investigación, básicamente de corte cualitativo, que comparten modos característicos de abordar los problemas y el diseño de ambientes educativos innovadores. La finalidad de los estudios de diseño es analizar, para comprender y mejorar, los procesos de enseñanza y aprendizaje en contexto mediante el diseño y estudio sistemático de formas particulares de aprendizaje, estrategias y herramientas de enseñanza (Molina, Castro, Molina y Castro 2011). Se han planteado diferentes propuestas acerca de cómo poner en práctica este tipo de metodologías. No obstante, existe cierto acuerdo en considerar tres fases importantes en un estudio de diseño cada una de las cuales puede conllevar distintos procedimientos metodológicos (Bannan-Ritland 2003). Así, Rinaudo y Donolo (2010) señalan las siguientes tres fases centrales del proceso investigativo: a) fase de preparación o de diseño; b) fase de implementación; c) fase de análisis retrospectivo. Con su aplicación al desarrollo de SEAs, se pretende obtener un «producto», la propia SEA, muy fundamentado desde el punto de vista teórico, ya que su diseño está basado en la investigación previa y, a su vez, se ha implementado y evaluado en la práctica mediante un proceso iterativo y cíclico que puede ser replicado en diferentes situaciones (Psillos y Kariotoglou 2016).

Utilizando los planteamientos de la IBD, el equipo investigador, los dos autores del artículo, diseñaron conjuntamente el proceso de investigación y las diferentes versiones de la SEA. El primer autor, profesor de Física y Química de Educación Secundaria con amplia experiencia docente, ha actuado también como implementador, asumiendo así el rol de profesor investigador en el aula (Porlán 1987). Se trata, por tanto, de un diseño colaborativo ya que en este tipo de investigación es importante que el profesorado tiene participe en la elección del contexto (Fensham 2009), así como en el diseño de la SEA, su implementación y evaluación.

\section{Presupuestos de partida}

Los planteamientos de diseño que se siguen en esta investigación toman ideas provenientes de diferentes modelos propuestos en la literatura, en particular, del modelo de reconstrucción educativa de Duit, Gropengießer y Kattmann (2005) y del modelo de diseño guiado por objetivos de aprendizaje de Krajcik, McNeill y Reiser (2008).

Esta investigación pretende, como se ha indicado antes, integrar en el seno de una SEA aspectos que provienen de campos muy diferentes. Se fundamenta en los enfoques de enseñanza en contexto y, particularmente, en las ideas de contexto como un evento concreto que toma sentido en su marco social (Gilbert 2006). También se pretende atender a los planteamientos de los currículos en nuestro país (MECD 2013) que demandan la integración 
del aprendizaje de contenidos científicos con el desarrollo de competencias y que requieren de nuevos planteamientos sobre cómo integrarlo en propuestas didácticas concretas (Franco, Blanco y España 2014 y 2017). Partiendo de estas ideas se está desarrollando un enfoque didáctico que se centra en la toma de decisiones sobre problemas de la vida diaria con la finalidad de desarrollar competencias científicas (Blanco, España, Franco y Rodríguez Mora 2018). En esta investigación el problema abordado es el fenómeno del consumo de agua envasada, que en la actualidad está dando lugar a una serie de controversias, tal y cómo se ha indicado en el apartado de fundamentos teóricos.

Para llevar a cabo la integración de estos aspectos se han formulado unos principios de diseño, entendidos en esta investigación como los pilares de apoyo de las tareas de diseño que sustenten (empírica, teórica o plausiblemente) el fomento de la finalidades y objetivos que se pretenden alcanzar (Euler 2017; Muñoz, Franco y España 2020). Estos principios concretan aspectos relevantes que las investigaciones sobre la enseñanza basada en el contexto han puesto de manifiesto y atienden a los requerimientos de los currículos sobre el desarrollo de competencias. Estos principios son:

a) La SEA tiene que posibilitar que los estudiantes construyan conocimientos científicos a la vez que aprenden aspectos relevantes sobre el problema tratado (aprender sobre el contexto y aprender ciencias a partir del contexto). Los problemas abordados y los conocimientos son igualmente importantes y ambos constituyen objetos de aprendizaje (Marchán y Sanmartí 2015) y, por ello, las relaciones entre ambos deben ser el eje para la estructuración de la SEA.

b) El tratamiento del problema debe desafiar a los estudiantes, ayudándoles a que pongan en juego sus ideas y creencias iniciales sobre el consumo de agua de bebida y a tomar decisiones razonadas y responsables sobre este consumo. Así, el uso de preguntas constituye un aspecto importante para orientar el aprendizaje de los estudiantes y, por ello, debe guiar la estructuración y la secuenciación de las actividades y tareas de la SEA (Edelson 2001; Krajick, McNeill y Reiser 2008).

c) La SEA tiene que promover la transferencia de los conocimientos científicos que los estudiantes van aprendiendo, para evitar que queden anclados y solo sean útiles en el contexto abordado (Gilbert, Bulte y Pilot 2011; Marchán y Sanmartí 2015)

d) Las actividades de la SEA tienen que ofrecer oportunidades para que los estudiantes desarrollen las competencias científicas. Para ello, es importante hacer explícitas en el diseño la vinculación de cada una de las actividades de enseñanza-aprendizaje con los aspectos concretos de las competencias que demandan (Franco, Blanco y España 2014 y 2017).

Tomando en consideración estos planteamientos, a continuación, se describen los aspectos más destacados del proceso de diseño y aplicación de una SEA sobre el consumo de agua envasada.

\section{Fase de preparación}

En esta primera fase se llevaron a cabo los estudios preliminares que se indican en la figura 1. 


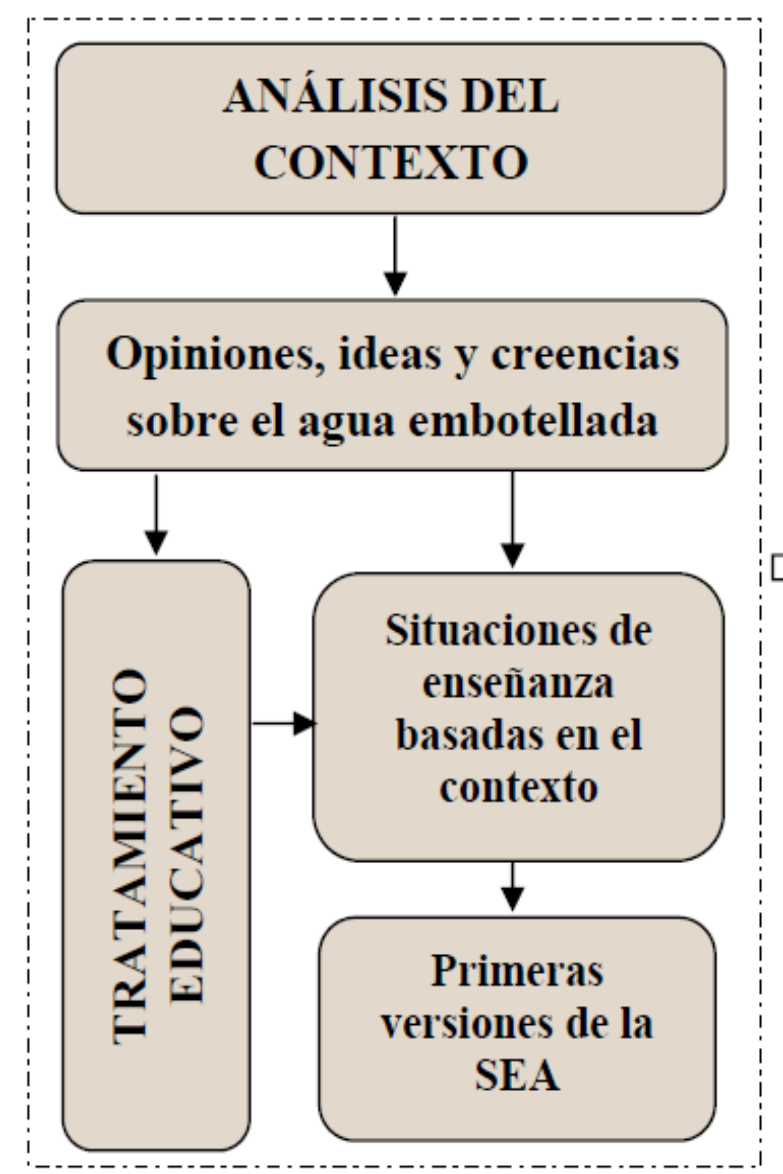

Figura 1. Descripción de los estudios preliminares para el desarrollo de la SEA sobre el consumo de agua envasada. Tomada de Rodríguez Mora (2016).

Esta fase estaba constituida por las siguientes tareas:

Tarea 1. Se analizaron los argumentos educativos más relevantes que justifican y fundamentan la elección del «consumo de agua de bebida envasada» como posible contexto educativo útil y adecuado. Así, se puso de manifiesto que se trata de un producto de consumo masivo en nuestro país y que forma parte de la vida diaria de los estudiantes. Su consumo va ligado a una serie de opiniones y creencias no siempre acordes con el punto de vista científico, y que pueden requerir de un trabajo específico en el aula. Por ello, su tratamiento en el aula permite acercar al alumnado a situaciones reales de su entorno y da prioridad a un conocimiento más real e integrado (ciencia en contexto). Igualmente, se puede utilizar como núcleo para abordar gran cantidad de conocimientos químicos (nociones de compuesto químico, mezcla y disolución, concentración, etc.), físicos (magnitudes físicas, cambio de unidades) y biológicogeológicos (tipos de microorganismos, contaminación, origen del agua, acuíferos), lo que permite establecer una adecuada relación de esta temática con distintos contenidos del currículum oficial.

Tarea 2. Se exploraron los puntos de vista más importantes que manifestaban 261 estudiantes de diferentes perfiles y niveles educativos $\left(3^{\circ}\right.$ y $4^{\circ} \mathrm{ESO}, 2^{\circ}$ Bachillerato y primer curso de Magisterio) (Rodríguez Mora y Blanco 2009 y 2012) con la finalidad de realizar un inventario de sus opiniones, ideas y creencias sobre el agua de bebida envasada y el agua del grifo. Entre las ideas y creencias más relevantes, encontradas en todas las muestras de estudiantes, se encuentran algunas relacionadas con la composición y seguridad del agua que bebemos: el 
agua envasada es más sana, saludable y segura que el agua del grifo; «es malo» consumir agua del grifo por la cal, porque no está limpia, no está controlada, etc.

En definitiva, se puso de manifiesto una percepción negativa del agua de consumo público en muchos de los participantes frente al agua envasada, que pasa a convertirse en una alternativa de superior calidad, aspectos sin fundamento científico de acuerdo con distintos informes técnicos existentes (Bullers 2002; Ferrier 2001). Entendíamos que la SEA debía, por tanto, contribuir a hacer conscientes a los estudiantes de estas creencias sobre el agua de abastecimiento público, a posibilitar su confrontación con ideas científicas, y a ayudarles a tomar decisiones razonadas y responsables sobre el consumo de agua de bebida (principio de diseño «b»).

Tarea 3. Se identificaron y seleccionaron posibles situaciones de enseñanza relacionadas con el contexto del agua envasada que podían ser más relevantes para los estudiantes, y que podrían formar parte de nuestra propuesta didáctica: la relación de su consumo con la salud, el agua como mercancía: el negocio del agua envasada, la moda del consumo de agua envasada como fenómeno social y los problemas ambientales asociados a un consumo masivo.

\section{Ciclos de diseño, implementación y de revisión}

De acuerdo con los planteamientos de la IBD, se han llevado a cabo tres ciclos de diseño, implementación y análisis que se recogen en la figura 2.

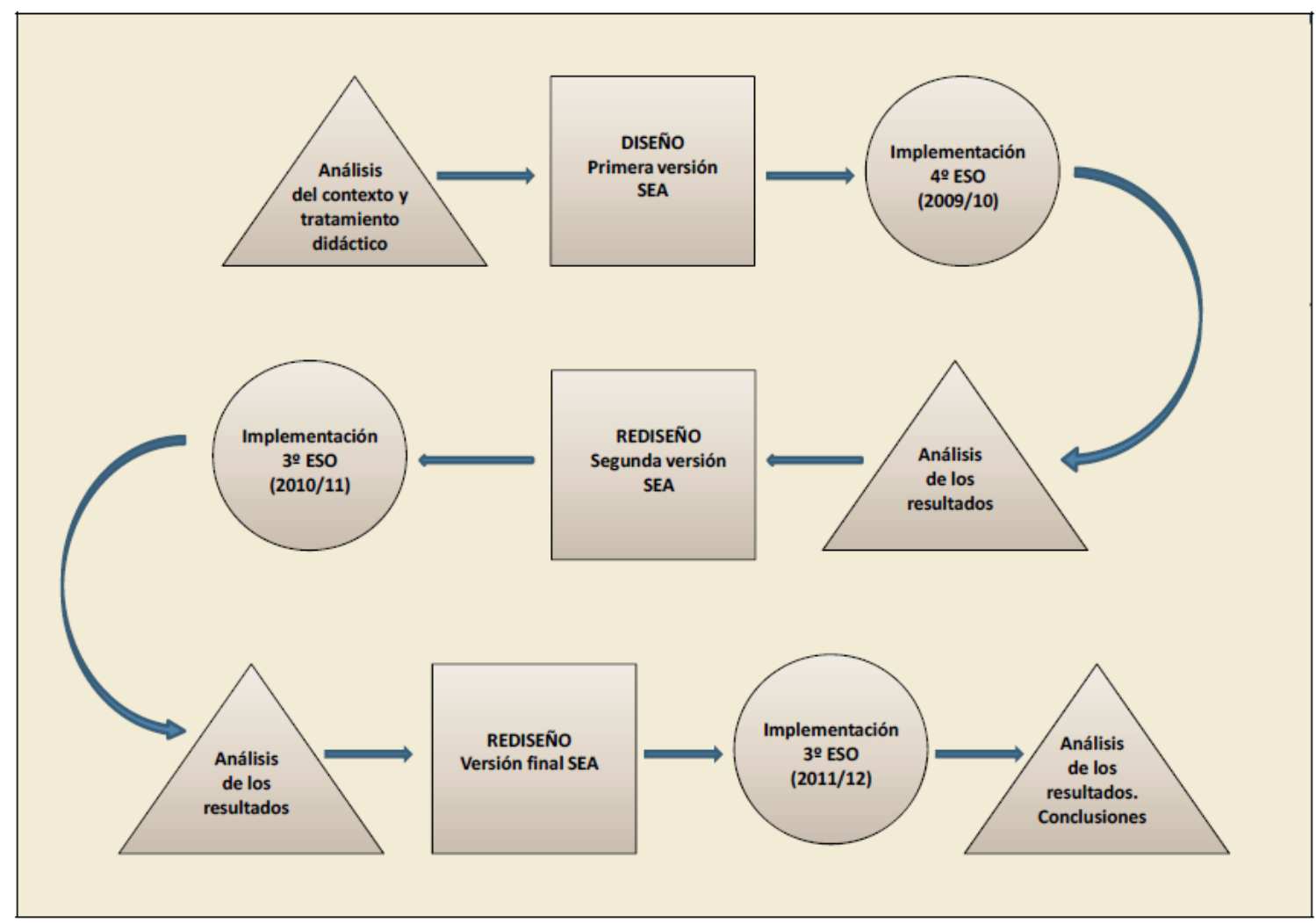

Figura 2. Ciclos iterativos de diseño, implementación y análisis realizados en esta investigación. Tomada de Rodríguez Mora (2016).

La elección de los niveles educativos para la puesta en práctica de las distintas versiones de la SEA fue intencional. Así, se creyó oportuno que el primer diseño de la SEA fuera implementado con estudiantes de $4^{\circ}$ ESO (opción científica) por varios motivos. En primer lugar, la materia de Física y Química en este curso tiene carácter optativo lo que suele traducirse en un mejor rendimiento académico y en un mayor interés de los estudiantes hacia 
dicha disciplina. En segundo lugar, este alumnado había recibido una formación más amplia en ciencias, y en concreto, sobre las disoluciones como sistemas materiales y los procesos de disolución, por lo que se consideró de interés analizar cómo los aplicaban al contexto elegido. Puesto que el estudio de las disoluciones en el momento de realizar la investigación solía abordarse en la materia de Física y Química, de carácter obligatorio, en $3^{\circ} \mathrm{ESO}$, se consideró la necesidad de presentar una segunda versión de nuestra SEA dirigida a este curso, con el objeto de analizar de qué manera podían integrarse los aspectos del contexto del agua de bebida con la construcción de conocimiento químico en torno al tópico de las disoluciones.

Algunos de los datos relevantes de la implementación de las tres versiones de la SEA (número de participantes y número de sesiones) se muestran en la tabla 1.

Tabla 1. Descripción de los participantes en la implementación de las distintas versiones de la SEA y del número de sesiones de clase utilizadas.

\begin{tabular}{|c|c|c|c|c|}
\hline VERSIÓN & $\begin{array}{c}\text { CURSO } \\
\text { ACADÉMICO }\end{array}$ & NIVEL EDUCATIVO & $\begin{array}{c}\text { NÚM. DE } \\
\text { SESIONES }\end{array}$ & $\begin{array}{c}\text { NÚM. DE } \\
\text { ESTUDIANTES }\end{array}$ \\
\hline SEA1 & $2009 / 2010$ & $4^{\circ}$ ESO (ciencias) & 4 & 23 \\
\hline SEA2.1 & $2010 / 2011$ & $3^{\circ}$ ESO & 7 & 20 \\
\hline SEA2.2 & $2011 / 2012$ & $3^{\circ}$ ESO & 13 & 25 \\
\hline
\end{tabular}

La descripción del desarrollo de la puesta en práctica de las distintas versiones de la SEA y la recolección de datos durante estas etapas del proceso investigativo, se realizan bajo el enfoque cualitativo de los estudios de caso (Yin 1989). La implementación de las tres propuestas didácticas se llevó a cabo en las condiciones normales de aula, mediante sesiones de 60 minutos de clase. Para el seguimiento de las distintas fases del desarrollo de la SEA se utilizaron, con carácter complementario, múltiples fuentes de datos (Rodríguez Mora 2016): valoración de los diseños por parte de experto, grabaciones en audio y vídeo de las clases, producciones escritas de los estudiantes, notas y diarios de clase del profesor, cuestionario de evaluación de los aprendizajes (Rodríguez Mora y Blanco 2016), cuestionarios de valoración, así como el informe sobre la puesta en práctica de la SEA2.2.

\section{Primer ciclo de diseño, implementación y revisión}

En el marco de esta investigación el interés de la SEA1 se centraba en avanzar hacia la integración de distintos aspectos del contexto del consumo de agua envasada y cómo presentar estos contenidos a los estudiantes (principios de diseño «a» y «b»). Así, se articulaba en torno a unos pocos interrogantes centrales que, girando en torno al agua de bebida envasada, guiaron su diseño y desarrollo: a) ¿necesitamos consumir agua envasada?; b) ¿es mejor el agua envasada que el agua del grifo? y c) ¿plantea problemas medioambientales el consumo de agua envasada?

La coherencia interna de la SEA1 fue valorada positivamente por cuatro profesores, de la especialidad de Física y Química de Educación Secundaria y de Didáctica de las Ciencias Experimentales, que actuaron en calidad de expertos y cuyas sugerencias y propuestas de mejora fueron incorporadas a la misma antes de su implementación en el aula. De esta manera, se mejoraron los enunciados de algunas de las actividades, se hicieron ciertos cambios en la secuenciación de los contenidos y se redujo la extensión de las actividades relacionadas con los problemas medioambientales asociados al consumo de agua envasada para facilitar su puesta en práctica.

Se realizó un ensayo en $4^{\circ}$ ESO (tabla 1) centrado en el problema del consumo de agua envasada, entendiendo esta versión como contexto de aplicación de conocimientos científicos. Esta primera experimentación también tenía la finalidad de servir de ensayo al profesor, ya que 
constituía su primera experiencia docente con este enfoque. Igualmente debemos indicar que era la primera vez que el grupo trabajaba «un tema así», en el que tenía que aplicar en el contexto del agua de bebida envasada conocimientos científicos que habían estudiado en el curso anterior, tales como caracterización de sistemas materiales, tipos de mezclas, propiedades básicas de las disoluciones, cálculos sobre composición cuantitativa de una disolución, etc.

\section{Segundo ciclo de diseño, implementación y revisión}

En la SEA2.1 se abordó la integración entre los conocimientos científicos y el problema tratado (principio de diseño «a»), entendiendo que esta versión de la SEA iba constituir un contexto de construcción de conocimientos y no de aplicación como lo era la primera versión. Para ello fue necesario incluir nuevas actividades que utilizaban el consumo de agua envasada como contexto para la construcción de conocimiento químico relacionado con las disoluciones.

Igualmente, se avanzó en la vinculación del desarrollo de competencias científicas y las actividades (principio de diseño «d»). Se realizó un análisis competencial para identificar qué aspectos concretos de las competencias científicas demandaban cada una de las actividades que se incluía en la SEA (Franco, Blanco y España 2014). Para los fines de esta investigación, se adoptó el marco que propone PISA en ciencias 2006 (OECD 2006), sobre cómo definir las diferentes competencias científicas y qué dimensiones o elementos son necesarios para su adquisición y desempeño. Este enfoque resultaba, desde nuestro punto de vista, sencillo y funcional al mostrar una formulación bastante precisa y, por ello, más fácil de utilizar para un profesorado, que en general, se estaba iniciando en el uso de las competencias como referente para la práctica educativa y para la evaluación. Recordemos que PISA 2006 contemplaba tres tipos de competencias científicas: identificación de cuestiones científicas (I), explicación científica de fenómenos (E) y utilización de pruebas $(\mathrm{U})$.

A raíz de la implementación y revisión de la SEA1 se identificaron algunas ideas y propuestas más concretas para la mejora del diseño. A modo de ejemplo, se indican algunas: las actividades a realizar por el alumnado fueron agrupadas en tareas, cada una de las cuales abordaba una dimensión clave relacionada con el consumo de agua de bebida envasada, se incorporó a la secuencia un apartado de introducción que contextualizaba y justificaba el interés del tema elegido y se incluyó una tarea sobre el visionado de un vídeo publicitario de una conocida marca de agua envasada para analizar la influencia de la publicidad en el consumo de este producto, y se avanzó en la mejora del diseño y redacción de algunas actividades.

Como ejemplo de los cambios que se fueron realizando en el diseño y presentación de las actividades, se comenta la actividad sobre el concepto de pureza del agua envasada, para abordar una de las creencias más arraigadas de los estudiantes (Rodríguez Mora 2016). En la SEA1 este aspecto se trataba en una actividad inicial, de explicitación de ideas previas, en la que se llamaba a los estudiantes la atención sobre un anuncio publicitario de una marca de agua envasada en la que se indicaba que es «verdaderamente pura» y en la etiqueta que es «agua mineral, sana y extremadamente pura» (figura 3). Se cuestionaba a los estudiantes si verdaderamente es «pura» el agua envasada y se les pedía manifestar verbalmente sus ideas al respecto. Después de la puesta en común se les animaba a implicarse en las actividades de la SEA para encontrar respuestas a esta pregunta. 


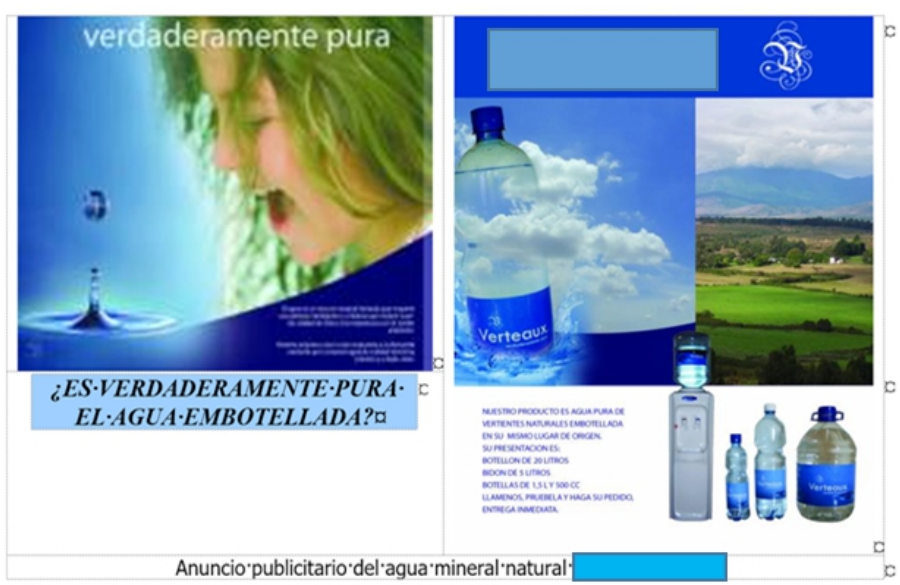

Figura 3. Imagen incluida en la actividad sobre la pureza del agua envasada en la SEA1. Tomada de Rodríguez Mora (2016).

En la SEA2.1 el concepto de pureza del agua envasada recibió una mayor atención, ya que los estudiantes que la recibieron eran de $3^{\circ}$ de ESO y en el seno de esta SEA estudiaban por primera vez en la asignatura de Física y Química los contenidos relativos a sistemas materiales y disoluciones. Se pretendía, por tanto, construir con ellos el concepto químico de sustancia pura y que lo diferenciaran de la idea de «pura» en la vida diaria. Incluyéndose también en el inicio de la secuencia se planteó, en esta versión, como una actividad de argumentación (figura 4), en particular para tratar con los estudiantes la necesidad de basarnos en pruebas (una de las competencias científicas en PISA 2006). Los estudiantes la cumplimentaban de forma individual y posteriormente se llevaba a cabo una puesta en común.

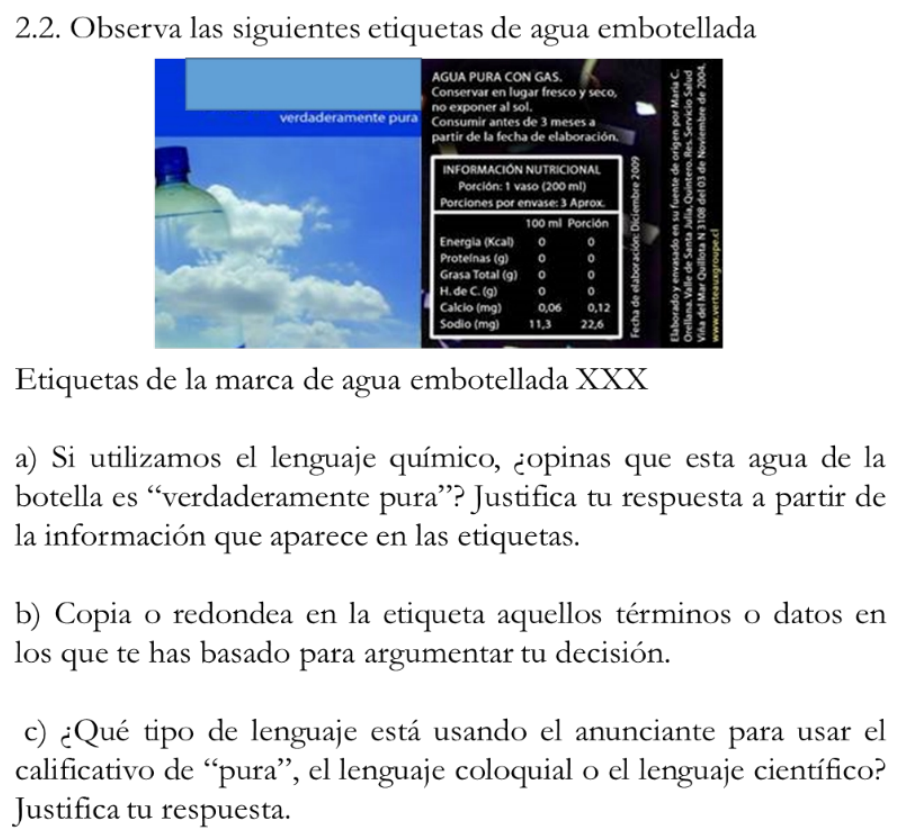

Etiquetas de la marca de agua embotellada XXX

a) Si utilizamos el lenguaje químico, ¿opinas que esta agua de la botella es "verdaderamente pura"? Justifica tu respuesta a partir de la información que aparece en las etiquetas.

b) Copia o redondea en la etiqueta aquellos términos o datos en los que te has basado para argumentar tu decisión.

c) ¿Qué tipo de lenguaje está usando el anunciante para usar el calificativo de "pura", el lenguaje coloquial o el lenguaje científico? Justifica tu respuesta.

Figura 4. Actividad sobre la pureza del agua envasada incluida en la SEA2.1. Tomada de Rodríguez Mora (2016).

El desarrollo de la SEA2.1 en $3^{\circ}$ ESO planteaba el hándicap asociado a la escasa carga lectiva de la materia de Física y Química, con solo dos horas semanales. Para evitar una dilatación excesiva en el tiempo se consideró necesario suprimir el tratamiento de alguno de los interrogantes centrales que habíamos contemplado en la SEA1. De acuerdo al catálogo de ideas y creencias manifestado por los estudiantes en los estudios preliminares pareció más oportuno suprimir la problemática relacionada con las repercusiones medioambientales del 
agua envasada, al aparecer con menor frecuencia en las respuestas de los estudiantes. De esta manera, se conviene para la SEA2.1, desarrollar únicamente los dos siguientes interrogantes clave: ¿es necesario consumir agua envasada? y ¿es mejor el agua envasada que el agua del grifo? (principio de diseño «b»).

Tras la segunda implementación y consecuente revisión se propusieron algunas modificaciones en la SEA2.1, tanto en su organización y estructura como en su contenido. Algunas de las más relevantes se indican a continuación: se incluye, para cada actividad, su aportación competencial de acuerdo con la propuesta que realiza PISA (2006), se incluyen algunas actividades prácticas sobre métodos de separación de mezclas, se profundiza en la caracterización química del agua de bebida como sistema químico material, así como en el uso y significado del término pureza, se incluye un cuestionario para la valoración de la propuesta por parte de los estudiantes, etc.

\section{Tercer ciclo de diseño, implementación y revisión}

Para la SEA2.2, ya adaptada a estudiantes de $3^{\circ}$ de ESO, se siguió trabajando en la mejora y formalización del diseño, tomando en consideración los cuatro principios de diseño formulados. Así, se adoptaron las etapas contempladas en la propuesta de Blanco, Franco y España (2016), que se estaba desarrollando en marco del proyecto al que pertenece esta investigación:

1. Elección del contexto de enseñanza y definición del problema.

2. Planteamiento de interrogantes en torno a los que girará la SEA.

3. Elaboración de los objetivos de aprendizaje.

4. Selección de los conocimientos, actitudes y valores.

5. Elaboración de la secuencia de actividades de enseñanza.

6. Diseño de la evaluación del aprendizaje de los alumnos.

Se llevó a cabo un nuevo análisis competencial de las actividades (principio de diseño «d»), se incluyeron nuevas actividades para mejorar la integración de los contenidos científicos y el problema tratado (principio de diseño «a») y se diseñaron actividades específicas para ayudar a los estudiantes a transferir los conocimientos sobre mezclas y disoluciones a otros contextos (alimentación, productos de higiene y de limpieza) (principio de diseño «c»).

Con respecto al seguimiento de la implementación de la SEA2.2 y con objeto de mejorar la calidad de los datos obtenidos se grabó en vídeo el desarrollo de las sesiones de clase, con el fin de poder realizar un análisis más exhaustivo de su desarrollo en el aula. De la misma manera, pareció conveniente no limitar las anotaciones del profesor, sobre el seguimiento de la puesta en práctica, al cumplimiento de una plantilla de tipo cerrado (lo que se hizo en los dos primeros ciclos), prefiriendo desarrollar un diario de clase «menos encorsetado». Con los datos obtenidos con estos instrumentos se confeccionó un amplio y detallado informe de la puesta en práctica que puede consultarse en Rodríguez Mora (2016).

\section{Resultados}

La presentación exhaustiva tanto del diseño como de los resultados de una IBD suele requerir más espacio del que habitualmente se dispone para un artículo (Guisasola, Zuza, Ametller, y Gutiérrez-Berraondo 2017). Así, en este apartado nos centraremos en resaltar solo los aspectos más relevantes de los resultados obtenidos. 


\section{La SEA sobre el consumo de agua envasada}

Como se ha indicado, la IBD en torno al desarrollo de SEAs presenta un carácter dual (Guisasola y Oliva 2020) y uno de sus resultados es el propio «artefacto didáctico» que ha sido objeto de investigación. En este caso, se ha generado un producto en forma de una SEA sobre el consumo de agua envasada que ha sido investigada y adaptada empíricamente al razonamiento de los estudiantes de $3^{\circ}$ de la ESO (Méheut y Psillos 2004).

Como resultado del proceso de diseño, implementación y análisis descrito en el apartado anterior, se llegó a una versión de la SEA (SEA2.2) que ha permitido integrar un buen número de elementos provenientes de diversos campos: a) aspectos relevantes sobre el consumo de agua de bebida (del grifo y envasada), esto es, aprender sobre el contexto; b) algunas ideas y creencias significativas sobre el consumo de agua de bebida, al objeto de contrastarlas con el conocimiento químico; c) contenidos científicos necesarios para comprender adecuadamente estos aspectos (aprender con el contexto) (tabla 2); y d) el desarrollo de competencias científicas.

Tabla 2. Contenidos científicos y contextuales tratados en la SEA2.2.

\begin{tabular}{|c|c|}
\hline CONTENIDO CIENTÍFICO & CONTENIDO CONTEXTUAL \\
\hline $\begin{array}{l}\text { - } \quad \text { Sustancias puras y mezclas de sustancias. } \\
\text { - } \text { en el ámbito de la vida cotidiana. } \\
\text { - Mezclas homogéneas y heterogéneas. } \\
\text { - Las disoluciones como mezclas homogéneas. } \\
\text { - Reversibilidad del proceso de disolución. } \\
\text { - Técnicas de separación física de los componentes } \\
\text { - } \text { de una disolución. } \\
\text { Presencia de las disoluciones en nuestra vida } \\
\text { diaria: en alimentos, productos de higiene, de } \\
\text { limpieza, etc. } \\
\text { - Modelos científicos para explicar el proceso de } \\
\text { disolución. } \\
\text { Factores de los que depende la composición de } \\
\text { una disolución. } \\
\text { Concentración. Expresión de la concentración en } \\
\text { masa en la unidad gramos de soluto por litro de } \\
\text { disolución. }\end{array}$ & $\begin{array}{l}\text { - El agua envasada no es un material químicamente } \\
\text { puro. } \\
\text { - El agua envasada y el agua del grifo como } \\
\text { - ejemplos de disoluciones. } \\
\text { - Los «iones fundamentales» en el agua de bebida. } \\
\text { - } \text { giferencias en la composición entre el agua del } \\
\text { - La seguridad en el agua de bebida. } \\
\text { - Controles sanitarios del agua de bebida (envasada } \\
\text { - } \quad \text { del grifo). } \\
\text { - } \text { envanesada. } \\
\text { Ventajas e inconvenientes que tiene el consumo } \\
\text { de agua de bebida envasada sobre la del grifo. }\end{array}$ \\
\hline
\end{tabular}

Una versión resumida de la SEA2.2 está disponible en Rodríguez Mora y Blanco (2016) y una descripción completa y detallada en Rodríguez Mora (2016). En este último trabajo también se pueden encontrar las pruebas utilizadas para concluir sobre su adecuación a los objetivos que se proponen.

Para analizar la adecuación de la SEA a sus objetivos, se utilizaron como instrumentos de toma de datos un cuestionario de evaluación del aprendizaje, un cuestionario de valoración de la experiencia por parte de los estudiantes y el informe de la puesta en práctica de la SEA2.2 (Rodríguez Mora 2016). De ellos se desprende que los estudiantes manifestaron sentirse interesados o muy interesados por diferentes aspectos de la temática tratada en dicha secuencia didáctica. Igualmente, consideraron que se habían sentido implicados o muy implicados durante su desarrollo en el aula. Estos resultados se observaron en las dos experiencias con estudiantes de $3^{\circ} \mathrm{ESO}$, lo que parece indicar que no se trata de una cuestión coyuntural. Por tanto, el interés y la implicación de los estudiantes parecen apuntar a la adecuación de este contexto para plantear situaciones relevantes de enseñanza y aprendizaje en el aula de ciencias. Esta valoración de los estudiantes coincide, con algunas matizaciones, con 
la valoración que del interés y la implicación de los estudiantes fue hecha por el profesor, en comparación con las clases más habituales de Física y Química, quizá por la «sensación de utilidad» manifestada por los propios estudiantes.

El desarrollo en el aula de la SEA2.2 ha ayudado a un buen número de estudiantes a mejorar su percepción inicial sobre el agua del grifo y sus efectos en el organismo. Aunque su sabor siga siendo considerado «malo» por buena parte de los estudiantes, en aspectos como la seguridad o la confianza hacia su consumo se ha producido un claro avance con respecto a los estudios anteriores (Rodríguez Mora 2006). En otras palabras, la gran mayoría de estudiantes estimó conveniente el consumo de agua de bebida envasada por su mejor sabor o comodidad de uso, pero no por razones relacionadas con un deficiente control o vigilancia sanitaria del agua del grifo.

Sobre el aprendizaje de conocimientos químicos, y teniendo en cuenta los que se han tratado en la SEA2.2 (tabla 2), los resultados obtenidos muestran diversas situaciones:

a) Los estudiantes diferenciaron adecuadamente los conceptos de mezcla y sustancia pura, en relación con la caracterización química del agua de bebida (del grifo o envasada).

b) Solo un reducido número de ellos utilizó con precisión el término «pureza» diferenciando claramente sus acepciones en los ámbitos de la química y de la vida cotidiana.

c) La mayoría presentó alguna dificultad para caracterizar «el agua del grifo» como un ejemplo de disolución, porque decían que «se veía la cal blanca del agua» (Rodríguez Mora y Blanco 2012).

d) Evidenciaron más dificultad para utilizar el concepto de interacción que el de movimiento a la hora de explicar el proceso de disolución en términos de un modelo de partículas (Blanco y Prieto 1994).

e) Mostraron también algunas dificultades, ya recogidas en la literatura (Blanco, 2000) para manejar la relación inversa en el cálculo de concentraciones y concebir este concepto como una magnitud intensiva, en el sentido de que su valor no depende del volumen de una disolución considerada.

Los estudiantes mostraron diferente grado de desempeño en las tres competencias científicas durante el desarrollo de la secuencia. En términos generales, las tareas relacionadas con la identificación de cuestiones científicas y la utilización de pruebas, se mostraron más complejas que aquellas otras relacionadas con la explicación científica de fenómenos. Esto puede deberse a que estas últimas son las que más se suelen fomentar en la enseñanza habitual (Cañas, Martín y Nieda 2007), y a la escasa familiaridad de los estudiantes con tareas que demandaban los distintos aspectos incluidos en las dos primeras competencias, especialmente el reconocimiento de cuestiones susceptibles de ser investigadas científicamente (identificación de cuestiones científicas) y la identificación de los supuestos y las pruebas que subyacen a las conclusiones (utilización de pruebas científicas). No obstante, es necesario matizar esta comparación tomando en consideración la diferente naturaleza de cada una de estas competencias, y el hecho de que para su evaluación se han utilizado diferentes procedimientos y herramientas de análisis y evaluación (Rodríguez Mora y Blanco 2016).

\section{Aportación a la enseñanza en contexto para el desarrollo de competencias científicas}

También se ha resaltado, dentro del carácter dual de la IBD, su aportación a la teorización sobre la enseñanza, produciendo «teorías humildes» según Guisasola y Oliva (2020). En este caso, consideramos que este trabajo hace algunas aportaciones a la propia metodología de 
investigación utilizada y a la teorización sobre la enseñanza para el desarrollo de competencias científicas en el contexto de problemas de la vida diaria.

En primer lugar, es importante indicar que la investigación aporta un ejemplo más en apoyo de la metodología de IBD como un enfoque adecuado para el diseño y desarrollo de SEAs (Guisasola y Oliva 2020). Los tres ciclos de diseño-implementación-análisis de distintas versiones de la SEA, han posibilitado la integración paulatina del problema planteado con el desarrollo de competencias científicas y la construcción de conocimiento.

También hay que indicar que esta investigación aporta un ejemplo concreto de cómo entender el contexto como un problema de la vida diaria que tiene que ser tratado en su marco social (Gilbert 2006) y que tanto los conocimientos científicos como el propio problema tratado son importantes (principio de diseño «a») (Marchán y Sanmartí 2015). Igualmente, la investigación ha ayudado a construir y aplicar un esquema de diseño de propuestas didácticas para el desarrollo de competencias científicas (Blanco, Franco y España 2016). Este enfoque integra una determinada forma de entender o conceptualizar las competencias científicas con el tratamiento de determinados contenidos científicos importantes en el currículo escolar de la ESO, en el contexto de un problema o situación de la vida diaria y a cómo vincularlas con las actividades de enseñanza-aprendizaje (principio de diseño «d»).

Este trabajo también aporta pruebas de las ventajas de la enseñanza basada en el contexto (Bennet, Lubben y Hogart 2007). Se constata una percepción y valoración positivas de los estudiantes hacia el empleo de este enfoque frente a otros más centrados en la transmisión del conocimiento científico. Estos resultados se apreciaron en los tres ciclos de implementación. Un número significativo de estudiantes valoró positivamente la innovación aplicada, prefiriendo volver a trabajar «de esta manera» en un futuro; o que considerara mejores o más divertidas las clases durante el desarrollo de la propuesta en relación a las clases «normales».

\section{Consideraciones finales}

Como toda investigación, ésta presenta algunas limitaciones que tienen que ver tanto con su objeto de estudio (desarrollar una SEA) como con el enfoque metodológico utilizado (IBD).

Con respecto al diseño hay que tener en cuenta la dificultad inherente que implica integrar en una misma SEA cuestiones tan diferentes: abordar un problema de la vida diaria, la construcción de conocimiento científico y el desarrollo de competencias científicas (principios de diseño «a» $\mathrm{y}$ 《d»). Habitualmente son problemas que suelen tratarse en la investigación didáctica de forma separada, al considerarse que cada uno constituye en sí mismo un tipo de investigación con metodologías o enfoques propios. Así, nos encontramos con el problema, muy resaltado en la bibliografía, de cómo integrar la enseñanza en contexto de la vida diaria con el aprendizaje de conocimientos científicos (Kortland 2007). Por otro lado, nos encontramos con dos problemas quizá más novedosos en la investigación didáctica, como es la integración del aprendizaje de contenidos con el desarrollo de competencias (Pedrinaci 2012) y la búsqueda de opciones concretas para enseñar y evaluar competencias en la enseñanza de las ciencias (Sanmartí y Marchán 2014). Atender esta variedad de cuestiones hace muy complejo abordarlas todas a la vez en el diseño de una SEA, aunque se tenga previsto hacerlo. Por ello, en este estudio se han ido incorporando de forma gradual en las distintas versiones de la SEA, para que se pudiese ir completándolas y mejorándolas tras su confrontación con la práctica.

Debemos tener presente también las limitaciones asociadas al estudio del desarrollo de competencias como producto de intervenciones didácticas de corto alcance en el tiempo. Se entiende que el desarrollo de competencias por parte de los estudiantes requiere brindar un 
buen número de situaciones para su desempeño, lo que demanda práctica y tiempo (Zabala y Arnau 2007), sobre todo si los estudiantes con los que se trabaja, como sucedió en esta investigación, no estaban habituados a este enfoque.

Dada su naturaleza, con esta investigación no se persigue conseguir una generalización estadística de los resultados obtenidos. Debemos asumir que en las tres implementaciones se ha trabajado con reducidos números de estudiantes. Por tanto, no se plantea que tales muestras sean consideradas representativas de respectivos niveles educativos. No obstante, los resultados obtenidos con respecto a las ideas y creencias de los estudiantes sobre el consumo de agua de bebida envasada, y las razones para dicho consumo, se hallan en concordancia con la literatura consultada (Da Cruz 2006, Ferrier 2001, Royte 2008). Desde esta perspectiva bien podrían considerarse representativos del estado de opinión de la ciudadanía al respecto.

En esta investigación se ha puesto el foco de atención en la descripción y análisis del proceso seguido, y desde el enfoque cualitativo que la impregna, hemos atendido más al cómo y al porqué que al cuánto. Desde el posicionamiento teórico de la IBD nuestro interés se ha centrado en obtener información sobre el diseño de la propuesta didáctica, la toma de datos de su implementación y desarrollo en el aula y su evaluación destinada a proponer cambios para la mejora. En este sentido, podemos hablar de una generalización basada en pruebas empíricas que apoyan la teoría general (en este caso la IBD y el desarrollo de secuencias de enseñanza en contexto) que ha servido para desarrollar una SEA concreta que ha funcionado en la práctica.

Por otro lado, y dada la naturaleza de los estudios de caso (Yin 1989), los resultados obtenidos pueden no ser directamente transferibles a otras situaciones similares, aunque se considera que el grado en que se han descrito el diseño y la implementación de la SEA2.2, así como su disponibilidad completa (Rodríguez Mora 2016) pueden permitir que otros investigadores o profesores puedan hacerse una idea muy detallada de la misma, lo que ayudará a su utilización en otros contextos educativos. Así, la SEA2.2, fruto de esta investigación, no debe entenderse como un producto final y acabado, sino como una hipótesis de trabajo que, partiendo de un contexto educativo concreto, se plantea para ayudar a los profesores a desarrollar las competencias científicas de los estudiantes tratando el consumo de agua de bebida envasada.

La siguiente fase de esta investigación sería, por tanto, su difusión y transferencia a otros profesores y contextos educativos. En este sentido, hay que tener en cuenta las dificultades, ya conocidas, de los procesos de transferencia de los resultados de la investigación a la práctica educativa (Millar y Osborne 2009). Además de disponer de una SEA bien diseñada se considera necesario que el profesorado comparta las finalidades de la misma, comprenda su fundamento y disponga de los conocimientos y habilidades docentes necesarios para llevarla a la práctica (Millar 2006).

\section{Agradecimientos}

Este trabajo forma parte del proyecto de I+D de Excelencia «Desarrollo de competencias en problemas de la vida diaria mediante prácticas científicas de argumentación, indagación y modelización en enseñanza secundaria y universitaria» (EDU2017-82197-P), financiado por el Ministerio de Ciencia, Innovación y Universidades en 2017.

\section{Referencias}

Bannan-Ritland B. (2003) The role of design in research: the integrative learning design framework. Educational Researcher 32(1), 21-24.

Bennett J., Lubben F. y Hogarth S. (2007) Bringing science to life: a synthesis of the research evidence on the effects of context-based and STS approaches to science teaching. Science Education 91(3), 347-370. 
Blanco A. (2000) Implicaciones didácticas de los estudios sobre las concepciones de los alumnos. Las Disoluciones. En Aspectos didácticos de física y quimica (Quimica 9) (pp. 101143). Zaragoza: Universidad de Zaragoza.

Blanco A., España E. y Rodríguez Mora F. (2012) Contexto y competencia científica. Alambique. Didáctica de las Ciencias Experimentales 70, 9-18.

Blanco A., Franco A.J. y España E. (2016) A competence-based approach to the design of a teaching sequence about oral and dental health and hygiene: a case study. Journal of Biological Education 50(2), 196-206.

Blanco A., España E., Franco, A.J. y Rodríguez Mora F. (2018) Competencias y prácticas científicas en problemas de la vida diaria. Alambique. Didáctica de las Ciencias Experimentales 92, 45-51.

Blanco, A. y Prieto, T. (1994) Las Disoluciones, dificultades de aprendizaje y sugerencias para su enseñanza. Alambique. Didáctica de las ciencias experimentales 1, 125-131.

Blanco A. y Rodríguez Mora, F. (2008) «El consumo de agua de bebida envasada» como contexto para desarrollar propuestas de alfabetización científica. En R. Márques et al. (Coords.), Perspectivas Ciencia-Tecnología-Sociedad en la Innovación de la Educación en Ciencias. Actas del V Seminario Ibérico y I Seminario Iberoamericano en CTS en la Educación Científica, (pp. 279-283), Aveiro, Portugal.

Bullers A.C. (2002). Bottled water: better than the tap? FDA Consumer Magazine 36(4), 14-18.

Buty C., Tiberghien A. y Le Maréchal J. F. (2004) Learning hyphotheses and an associated tool to design and to analyse teaching-learning sequences. International Journal of Science Education 26(5), 579-604.

Caamaño A. (2005) Presentación de la monografía: Contextualizar la ciencia. Una necesidad en el nuevo currículo de ciencias. Alambique. Didáctica de las Ciencias Experimentales, 46, 58 .

Cañas A., Martín-Díaz M. y Nieda J. (2007) Competencia en el conocimiento y la interacción con el mundo físico. La competencia científica. Madrid: Alianza Editorial.

Couso D. (2011) Las secuencias didácticas en la enseñanza y el aprendizaje de las ciencias: modelos para su diseño y validación. En A. Caamaño (Coord.), Didáctica de la Física y la Quimica (pp 57-84). Barcelona: Graó.

Da Cruz J. (2006) Agua envasada: signo de nuestro tiempo. Observatorio de la Globalización 5, 1-6.

DBRC (2003) Design-based research: an emerging paradigm for educational inquiry. Educational Researcher 32(1), 5-8.

Duit, R., Gropengießer, H., y Kattmann, U. (2005). Towards science education research that is relevant for improving practice: The model of educational reconstruction. In H. Fisher (Ed.), Developing standards in research on science education ( $p$. 1-9). London: Taylor and Francis.

Edelson D.C. (2001) Learning-for-use: A framework for integrating content and process learning in the design of inquiry activities. Journal of Research in Science Teaching 38(3), 355-385.

Euler D. (2017) Design principles as bridge between scientific knowledge production and practice design. EDeR - Educational Design Research 1(1), 1-15. 
Fensham P. (2009) Real world contexts in PISA science: Implications for context-based science education. Journal of Research in Science Teaching,46(8), 884-896.

Ferrier C. (2001) Bottled water: understanding a social phenomenon. A Journal of the Human Environment 30(2), 118-140.

Franco A.J., Blanco A. y España E. (2014) El desarrollo de la competencia científica en una unidad didáctica sobre la salud bucodental. Diseño y análisis de tareas. Enseñanza de las Ciencias 32(3), 649-667.

Franco A.J., Blanco A. y España E. (2017) Diseño de actividades para el desarrollo de competencias científicas. Utilización del marco de PISA en un contexto relacionado con la salud. Revista Eureka sobre Enseñanza y Divulgación de las Ciencias 14(1), 38-53.

Gavidia, V. (2009) El agua en las enseñanzas mínimas de secundaria. En A. Moreno y C. López (Coords.), Agua y sostenibilidad: recursos, riegos y remedios (pp. 61-94). Madrid: Ministerio de Educación, Cultura y Deporte.

Gilbert J. (2006) On the nature of «context» in chemical education. International Journal of Science Education 28(9), 957-976.

Gilbert, J.; Bulte, A. y Pilot, A. (2011) Concept development and transfer in context based science education. International Journal of Science Education 33(11), 817-837.

Gleick P.H. y Cooley H.S. (2009) Energy implications of bottled water. Environmental Research Letters 4, 14009-14015.

Guisasola J., y Oliva J. M. (2020) Nueva sección especial de REurEDC sobre investigación basada en el diseño de secuencias de enseñanza-aprendizaje. Revista Eureka sobre Enseñanza y Divulgación de las Ciencias 17(3), 3001.

Guisasola J., Zuza K., Ametller J., y Gutierrez-Berraondo, J. (2017) Evaluating and redesigning teaching learning sequences at the introductory physics level. Physical Review Physics Education Research 13(2), 020139-1/14.

Jiménez V. y Otero J. (2012) Acceso y procesamiento de información sobre problemas científicos de relevancia social: limitaciones en la alfabetización científica de los ciudadanos. CTS: Revista Iberoamericana de Ciencia, Tecnología y Sociedad 7(20), 29-54.

Kortland J. (2007) Context-based science curricula: Exploring the didactical friction between context and science content. Paper presented at the ESERA 2007 Conference, Malmö, Sweden.

Krajcik J., McNeill K.L. y Reiser B.J. (2008) Learning-goals-driven design model: Developing curriculum materials that align with national standards and incorporate project-based pedagogy. Science Education 92(1), 1-32.

Marcen C. (2009) El agua, argumento educativo en la Educación Obligatoria y en el sistema social. Tesis doctoral. Zaragoza: Universidad de Zaragoza.

Marcén C. y Cuadrats J.M. (2012) Argumentos educativos para enseñar-aprender el agua en la enseñanza obligatoria. Serie Geográfica 18, 65-75.

Marchán I. y Sanmartí N. (2015) Criterios para el diseño de unidades didácticas contextualizadas: aplicación al aprendizaje de un modelo teórico para la estructura atómica. Educación Química 26(4), 267-274.

MEC (2006) Ley Orgánica 2/2006, de 3 de mayo, de Educación. BOE núm. 104 de 4 de mayo de 2006. 
MECD (2013) Ley Orgánica 8/2013, de 9 de diciembre, de Mejora de la Calidad Educativa. BOE núm. 295 de 10 de diciembre de 2013.

Méheut M. y Psillos D. (2004) Teaching-learning sequences: aims and tools for science education research. International Journal of Science Education 26(5), 515-535.

Millar R. (2006) Twenty first century science: implications from the design and implementation of a scientific literacy approach in school science. International Journal of Science Education 28(13), 1499-1521.

Millar R. y Osborne J. (2009) Research and practice: A complex relationship? En M.C. Shelley II, L.D Yore y B. Hand (Eds.), Quality research in literacy and science education. International perspectives and gold standards (pp.41-61). Dordrecht, Holand: Springer.

Molina M., Castro E., Molina J.L. y Castro Enrique (2011) Un acercamiento a la investigación de diseño a través de los experimentos de enseñanza. Enseñanza de las Ciencias 29(1), 75 88 .

Muñoz V. Franco A.J. y Blanco A. (2020) Integration of scientific practices into daily living contexts: a framework for the design of teaching-learning sequences, International Journal of Science Education, 42(15), 2574-2600.

OECD (2006) Assessing scientific, reading and mathematical literacy: A framework for PISA 2006. Paris, Francia: OECD.

Pedrinaci E. (2012) El ejercicio de una ciudadanía responsable exige disponer de cierta competencia científica. En E. Pedrinaci (Coord.) Once ideas clave. El desarrollo de la competencia científica (pp. 15-35). Barcelona: Graó.

Porlán R. (1987) El maestro como investigador en el aula. Investigar para conocer, conocer para enseñar. Investigación en la Escuela 1, 63-70.

Pryde L., Middlecamp C., Pienta N., Heltzel C. y Weawer E. (2005) Chemistry in context. Applying chemistry to society. Boston, Estados Unidos: McGraw-Hill.

Psillos D. y Kariotoglou P. (2016) Iterative desing of teaching-learning sequences. Switzerland: Springer Nature.

Rinaudo M. y Donolo D. (2010) Estudios de diseño. Una perspectiva prometedora en la investigación educativa. RED. Revista de Educación a Distancia, 22.

Rodríguez Mora F. (2016) El consumo de agua de bebida envasada como contexto para el desarrollo de competencias científicas. Un estudio de caso en tercer curso de la Educación Secundaria Obligatoria. Tesis Doctoral. Málaga: Universidad de Málaga. Disponible: https://riuma.uma.es/xmlui/handle/10630/13315

Rodríguez Mora F. y Blanco A. (2009) Conocimientos, hábitos, actitudes y creencias de estudiantes de Magisterio sobre el consumo de aguas de bebida envasadas. Enseñanza de las Ciencias, número extra. Actas del VIII Congreso Internacional sobre Investigación en la Didáctica de las Ciencias, 1890-1894.

Rodríguez Mora F. y Blanco A. (2012) Ideas y creencias de alumnos de educación secundaria sobre la presencia de «cal» en el agua de bebida. En G. Pinto y M. Martín (Eds.), Enseñanza y Divulgación de la Química y la Física (pp. 197-205). Madrid: Garceta Grupo Editorial.

Rodríguez Mora F., y Blanco A. (2016) Diseño y análisis de tareas de evaluación de competencias científicas en una unidad didáctica sobre el consumo de agua envasada 
para educación secundaria obligatoria. Revista Eureka sobre Enseñanza y Divulgación de las Ciencias 13(2), 279-300.

Royte E. (2008) Bottlemania. How water went on sale and why we bought it. New York: Bloomsbury.

Sanmartí N. (2008) ¿Què comporta desenvolupar la competéncia científica? Guix, 344, 11-16.

Sanmartí N. y Marchán I. (2014) ¿Cómo elaborar una prueba de evaluación escrita? Alambique. Didáctica de las Ciencias Experimentales 78, 1-10.

Shotyk W., Krachler M. y Chen B. (2006) Contamination of Canadian and European bottled waters with antimony from PET containers. Journal of Environmental Monitoring 8, 288292.

Tekkumru-Kisa M., Stein M.K. y Schunn C. (2015) A Framework for Analyzing Cognitive Demand and Content-Practices Integration: Task Analysis Guide in Science. Journal of Research in Science Teaching 52(5), 659-685.

Wilk R. (2006) Bottled water: the pure commodity in the age of branding. Journal of Consumer Culture 6, 303-325.

Yin R. (1989) Case Study Research. Design and methods. London, UK: SAGE Publications.

Zabala A. y Arnau L. (2007) 11 ideas clave. Cómo aprender y enseñar competencias. Barcelona: Graó. 